\title{
SALINIDADE NA EMERGÊNCIA E NO CRESCIMENTO INICIAL DE MULUNGU
}

\section{Elaine Cristina Alves da Silva ${ }^{1 *}$, Jenickson Rayron da Silva Costa ${ }^{2}$, Pétala Carvalho Ferreira da Costa ${ }^{3}$, Andreia Michelle Alves Cunha de Alcantara ${ }^{4}$, Cibele Alves dos Santos ${ }^{1}$, Rejane Jurema Mansur Custódio Nogueira ${ }^{5}$}

\author{
${ }^{1}$ Doutora em Biotecnologia, Universidade Federal Rural de Pernambuco (UFRPE), Rua Dom Manuel de Medeiros, s/n, Dois Irmãos - \\ Recife/PE - CEP: 52.171-900 \\ ${ }^{2}$ Graduando em Engenharia Florestal, Universidade Federal Rural do Semi Árido (UFERSA), Av. Francisco Mota, 572 - Bairro Costa e \\ Silva, Mossoró/RN - CEP: $59.625-900$ \\ ${ }^{3}$ Graduanda em Engenharia Florestal, Universidade Federal Rural de Pernambuco (UFRPE), Rua Dom Manuel de Medeiros, s/n, Dois \\ Irmãos - Recife/PE - CEP: 52.171-900 \\ ${ }^{4}$ Graduada em Ciências Biológicas, Universidade Federal Rural de Pernambuco (UFRPE), Rua Dom Manuel de Medeiros, s/n, Dois \\ Irmãos - Recife/PE - CEP: 52.171-900 \\ ${ }^{5}$ Profa. Titular, Universidade Federal Rural de Pernambuco (UFRPE), Rua Dom Manuel de Medeiros, s/n, Dois Irmãos - Recife/PE - \\ CEP: $52.171-900$
}

*Autor para correspondência: Elaine Cristina Alves da Silva, elainemanancial@gmail.com

RESUMO: Este trabalho teve como objetivo avaliar o efeito da salinidade na emergência e no crescimento inicial de plântulas de Mulungu (Erythrina velutina Willd.). 0 experimento foi desenvolvido em casa de vegetação na Universidade Federal Rural de Pernambuco. 0 delineamento experimental utilizado foi inteiramente casualizado, com cinco tratamentos e quatro repetições de 25 sementes cada. Os tratamentos salinos foram: 0, 25, 50, 75 e $100 \mathrm{mM}$ de $\mathrm{CaCl}_{2}$. Avaliou-se a porcentagem de emergência (\%E), índice de velocidade de emergência (IVE), tempo médio de emergência (TME) e velocidade média de emergência (VME). Ao final do experimento, mediuse a altura da parte aérea, o comprimento da raiz, altura total e o diâmetro da plântula, contabilizou-se o número de folhas e determinou-se o peso da matéria seca da parte aérea, raiz e total. Todas as variáveis avaliadas foram afetadas com 0 aumento do nível salino. A germinação e o crescimento das plântulas foram reduzidos em, aproximadamente, $96 \%$ e 100\%, respectivamente, para o nível mais salino (100 mM de $\mathrm{CaCl}_{2}$ ). Sementes de $E$. velutina tem sua germinação afetada a partir do nível de $50 \mathrm{mM}$ de $\mathrm{CaCl}_{2}$, contudo o desenvolvimento das plântulas é afetado a partir de $25 \mathrm{mM}$ de $\mathrm{CaCl}_{2}$.

PALAVRAS CHAVE: Erythrina velutina, estresse salino, $\mathrm{CaCl}_{2}$.

\section{SALINITY IN EMERGENCE AND INITIAL GROWTH OF MULUNGU}

ABSTRACT: This work aimed to evaluate the effect of salinity on emergence and initial growth of Mulungu (Erythrina velutina Willd.) seedlings. The experiment was carried out under greenhouse conditions at the Federal Rural University of Pernambuco. The experimental design was completely randomized, with five treatments and four replicates of 25 seeds each. Saline treatments were: $0,25,50,75$ and $100 \mathrm{mM} \mathrm{CaCl}_{2}$. The percentage of emergency (\% E), velocity index of emergency (IVE), mean time of emergency (TME) and mean velocity of emergence (EMV) were evaluated. At the end of the experiment, shoot height, root length, total height and seedling diameter were counted, the number of leaves was counted and the dry matter weight of shoot, root and total. All the variables evaluated were affected with the saline level increase. Germination and seedling growth were reduced by approximately $96 \%$ and $100 \%$, respectively, to the more saline level $\left(100 \mathrm{mM} \mathrm{CaCl}_{2}\right)$. Seeds of Erythrina velutina have their germination affected from the $50 \mathrm{mM} \mathrm{CaCl} 2$ level, however seedling development is affected from 25 $\mathrm{mM} \mathrm{CaCl}_{2}$.

KEY WORDS: Erythrina velutina, salt stress, $\mathrm{CaCl}_{2}$. 


\section{INTRODUÇÃO}

A Erythrina velutina Willd. (Fabaceae), conhecida vulgarmente como mulungu, é uma espécie secundária com dispersão irregular e descontínua (Gonçalves et al., 2014). A casca e as sementes de mulungu possuem eritrina, um alcaloide que atua no sistema nervoso, podendo causar paralisia e, ao ser macerada, a casca tem ação hipnótica e narcótica. Apesar da ausência de comprovações científicas quanto aos efeitos de seu uso e sua eficácia, adeptos da medicina popular utilizam a casca e os frutos como calmante, emoliente, anestésico local, odontálgico, sedativo, calmante de tosses e bronquites e, também, no tratamento de verminoses e hemorroidas (Carvalho, 2008).

O mulungu é uma planta ocorrente no semiárido, distribuido pelos estados do Piauí, Ceará Rio Grande do Norte, Paraíba, Pernambuco, Sergipe e Minas Gerais (Elhag e Abdalla, 2012). Várias áreas da Caatinga encontram-se degradadas pelos altos índices de sais, prejudicando a germinação e o desenvolvimento dos vegetais, por isso, a identificação da tolerância ou sensibilidade das espécies à salinidade é importante para indicar espécies com potencial para serem utilizadas em projetos de reflorestamento de áreas salinizadas (Vasconcelos et al., 2013).

Em regiões secas, como as regiões áridas $e$ semiáridas, a salinidade é uma situação que ocorre frequentemente, devido a fatores ambientais $\mathrm{e}$ antrópicos, como a baixa precipitação pluviométrica, altas temperaturas, além da drenagem deficiente e a utilização de água salinizada pelo homem na irrigação, o que favorece 0 acúmulo de sais no solo (Holanda et al., 2007).

A salinidade afeta negativamente as propriedades físicas e químicas do solo (Barros et al., 2004). Em relação às propriedades químicas, 0 aumento das concentrações de sais ocasiona a redução de sua fertilidade enquanto nas propriedades físicas do solo, provoca a desestruturação dos agregados do solo, aumento da densidade e redução das taxas de infiltração de água pelo excesso de íons sódicos (D'Almeida et al., 2005).

A germinação é um processo caracterizado pela retomada do crescimento embrionário após a embebição da água pela semente, e para que a mesma ocorra, é necessário que haja condições favoráveis. Fatores como a luz, a temperatura, a umidade e a presença de sais no substrato podem inibir ou retardar o processo germinativo (Barros et al., 2004; Marcos Filho, 2015).

O acúmulo de sais no solo é um fator de estresse para o vegetal, pois causa redução do seu potencial hídrico, dificultando assim a absorção de água pelas sementes, o que pode reduzir ou até mesmo inibir a germinação, uma vez que, além de reduzir o potencial osmótico, proporciona a ação dos íons sobre o protoplasma das células das sementes (D'Almeida et al., 2005). Embora a germinação ocorra, o aumento da salinidade dificulta a absorção de água pelas raízes das plântulas, o que prejudica no seu estabelecimento (Dias e Blanco, 2010).

Sendo assim, o presente trabalho teve como objetivo avaliar o efeito de diferentes concentrações salinas de $\mathrm{CaCl}_{2}$ na emergência e no desenvolvimento inicial de plântulas de mulungu.

\section{MATERIAL E MÉTODOS}

0 experimento foi desenvolvido em casa de vegetação na Universidade Federal Rural de Pernambuco, situada no município de Recife-PE. A essência florestal utilizada foi o mulungu, e os frutos coletados em matrizes no município de Petrolina-PE. Antes do semeio, as sementes foram escarificadas, na região oposta ao hilo, com 0 auxílio de uma lixa $n^{\circ} 80$, para superação da dormência.

0 delineamento estatístico utilizado foi 0 inteiramente casualizado, com cinco tratamentos e quatro repetições de 25 sementes cada. Constituíram os tratamentos as soluções salinas preparadas nas concentrações de 0, 25, 50, 75 e 100 mM, adicionandose cloreto de cálcio $\left(\mathrm{CaCl}_{2}\right)$ à água destilada, correspondendo às condutividades elétricas de 0,01 ; 3,$1 ; 5,52 ; 6,54$ e $8,20 \mathrm{dS} \cdot \mathrm{m}^{-1}$. As sementes foram semeadas em bandejas de polietileno de dimensões $18 \times 13 \times 4,5 \mathrm{~cm}$, preenchidas com areia lavada, e as irrigações realizadas uma vez ao dia, com um volume médio de $100 \mathrm{~mL}$ por repetição, aplicando as soluções salinas de acordo com as concentrações estabelecidas.

As características avaliadas foram: a porcentagem de emergência de plântulas, estabelecida com base na observação da emergência (\%E), diariamente após a semeadura até o $15^{\circ} \mathrm{dia}$, sendo consideradas como emergidas quando os cotilédones foram expostos na superfície do solo; 0 índice de 
velocidade de emergência (IVE), de acordo com Maguire (1962); o tempo médio de emergência (TME), segundo Labouriau (1983); a velocidade média de emergência (VME); a altura de plântulas e comprimento da raiz, realizada com 0 auxílio de régua graduada em milímetro, sendo os dados obtidos expressos em centímetros; o número de folha, pela contagem do número total de folhas por planta; 0 diâmetro, com 0 auxilio de um paquimetro digital; e a massa seca da parte aérea, obtida a partir de uma amostra de 10 plântulas da parcela, após secagem em estufa de circulação de ar forçado a $65{ }^{\circ} \mathrm{C}$ até obtenção de peso constante, com uso de balança analítica com precisão de $0,0001 \mathrm{~g}$.

Tabela 1. Resumo da análise de variância das características porcentagem de emergência ( $E$ \%), índice de velocidade de emergência (IVE), tempo médio de emergência (TME), velocidade média de emergência (VME), altura da plântula (AP), comprimento da raiz (CR), altura total (AT), número de folhas (NF) e diâmetro do caule (DC) de plântulas de mulungu (Erythrina velutina Willd.) submetidas a diferentes níveis de salinidade.

\begin{tabular}{|c|c|c|c|c|c|c|c|c|c|c|}
\hline \multirow{2}{*}{ FV } & \multicolumn{10}{|c|}{ Características avaliadas } \\
\hline & $G L$ & $E \%$ & IVE & TME & VME & $P A$ & $C R$ & AT & $N F$ & $D C$ \\
\hline Tratamento & 4 & $6237,20^{*}$ & $36,32^{*}$ & $11,01^{*}$ & $7,61^{*}$ & $149,4^{*}$ & $233,31^{*}$ & $277,64^{*}$ & $5,23^{*}$ & $10,20^{*}$ \\
\hline Erro & 15 & 36,80 & 0,23 & 0,16 & 0,16 & 0,82 & 0,32 & 1,57 & 0,02 & 0,06 \\
\hline Média & - & 48,40 & 3,34 & 2,32 & 1,11 & 7,22 & 5,35 & 12,57 & 0,83 & 2,04 \\
\hline CV $(\%)$ & - & 12,53 & 14,37 & 17,04 & 35,58 & 19,02 & 17,72 & 15,73 & 19,09 & 11,92 \\
\hline
\end{tabular}

GL - Graus de liberdade; CV- Coeficiente de variação; * e **- Significativo a 0,01 e a 0,05 de probabilidade, respectivamente, pelo teste de F.

A porcentagem de emergência de plântulas de Erythrina velutina foi afetada pela adição do $\mathrm{CaCl}_{2}$ com redução significativa a partir da concentração de $50 \mathrm{mM}$, onde a \%E foi de $56 \%$. Já nas concentrações de 75 e $100 \mathrm{mM}$ de $\mathrm{CaCl}_{2}$, a redução mais acentuada, 86 e 96\%, respectivamente em relação ao controle, evidenciando a sensibilidade da espécie aos referidos níveis de $\mathrm{CaCl}_{2}$ (Tabela 2).

Tabela 2. Valores médios da porcentagem de emergência (\%E), índice de velocidade de emergência (IVE), tempo médio de emergência (TME) e velocidade média de emergência (VME) de sementes de Erythrina velutina submetidas a diferentes tratamentos salinos.

\begin{tabular}{ccccc}
\hline Tratamentos salinos $\left(\mathrm{mM} \mathrm{de} \mathrm{CaCl}_{2}\right)$ & $\% E$ & IVE & TME (Dias) & VME $\left(\right.$ Plântula.dia $\left.{ }^{-1}\right)$ \\
\hline 0 & $90,0 \mathrm{a}$ & $6,70 \mathrm{a}$ & $3,60 \mathrm{a}$ & $0,27 \mathrm{c}$ \\
25 & $81,0 \mathrm{a}$ & $6,08 \mathrm{a}$ & $3,44 \mathrm{a}$ & $0,29 \mathrm{c}$ \\
50 & $56,0 \mathrm{~b}$ & $3,14 \mathrm{~b}$ & $3,50 \mathrm{a}$ & $0,29 \mathrm{c}$ \\
75 & $12,0 \mathrm{c}$ & $0,56 \mathrm{c}$ & $0,90 \mathrm{~b}$ & $3,22 \mathrm{~b}$ \\
100 & $3,0 \mathrm{c}$ & $0,21 \mathrm{c}$ & $0,15 \mathrm{~b}$ & $3,47 \mathrm{a}$ \\
\hline $\mathrm{CV}(\%)$ & 12,53 & 14,37 & 17,04 & 35,58 \\
\hline
\end{tabular}

*Médias seguidas da mesma letra na coluna, não diferem estatisticamente entre si pelo teste de Tukey a $5 \%$ de probabilidade. CV Coeficiente de variação.

Assim como a porcentagem de emergência, a redução do IVE de forma significativa teve início a partir de $50 \mathrm{mM}$ de $\mathrm{CaCl}_{2}(53 \%)$ e para os níveis de 75 e $100 \mathrm{mM}$ a redução foi de 92 e 97\%, respectivamente, em relação ao controle (Tabela 2).
Esta redução no índice de velocidade da emergência do mulungu pode ser devido à redução do potencial osmótico do substrato, o que afetou a disponibilidade de água a ser absorvida pelas sementes interferindo na germinação. 
Não houve diferença estatística para o TME e VME das sementes submetidas aos níveis de 0, 25 e 50 $\mathrm{mM}$ de $\mathrm{CaCl}_{2}$. A maior velocidade média de emergência (1,22 e 3,47 plântula.dia-1 ${ }^{-1}$ foi observada nos níveis de 75 e $100 \mathrm{mM}$ de $\mathrm{CaCl}_{2}$, devido à menor porcentagem de emergência que ocorreram nesses tratamentos, nos quatro primeiros dias de germinação, não havendo mais germinação, após esse período (Tabela 2).

Resultados semelhantes foram observados por Lima e Torres (2009), que ao estudarem o efeito da salinidade em sementes de Zizyphus joazeiro Mart, observaram que o aumento da concentração salina provocou redução na germinação. Assim como, Barreto et al., (2010), Ferreira et al. (2013) e Gordim et al. (2012), que ao estudarem as espécies Mimosa caesalpiniifolia, Cedrela odorata e Apeiba tibourbou, respectivamente, observaram efeito deletério da salinidade na germinação. 0 acúmulo de sais no substrato reduz o potencial hídrico deste, ocasionando a indisponibilidade de água para a semente, sendo assim, a água não é absorvida pela semente reduzindo a germinação (Holanda et al., 2007). A salinidade afeta a germinação, pois além de provocar um desbalanço osmótico, ocasiona toxicidade devido ao acúmulo dos íons no protoplasma (Lima et al., 2009).

A salinidade também afetou negativamente 0 crescimento de plântulas de $E$. velutina, ocasionando redução linear na altura da plântula, comprimento da raiz, altura total e produção de biomassa. 0 comprimento da parte aérea das plântulas que germinaram sob salinidade, teve redução variando de 45\% para o nível de $25 \mathrm{mM}$, até $99 \%$ para o nível de $100 \mathrm{mM}$ de $\mathrm{CaCl}_{2}$. $O$ comprimento da raiz também foi afetado significativamente desde a concentração de $25 \mathrm{mM}$, com uma redução de $54 \%$ e para os demais tratamentos (50 a $100 \mathrm{mM}$ ), a redução foi de $93 \%$, $97 \%$ e $98,5 \%$. A altura total também foi reduzida em $47,5 \%$ para as plântulas germinadas ao nível de $25 \mathrm{mM}$ de $\mathrm{CaCl}_{2}$, chegando a $99 \%$ para o nível de $100 \mathrm{mM}$ (Tabela 3).

Tabela 3. Valores médios da altura da parte aérea $(P A)$, comprimento da raiz $(C R)$, altura total $(A T)$, número de folhas (NF), diâmetro do colo (DC), peso da matéria seca da parte aérea (PMSPA), peso da matéria seca da raiz (PMSR) e peso da matéria seca total (PMST) de plântulas de Erythrina velutina germinadas sob diferentes tratamentos salinos.

\begin{tabular}{ccccccccc}
\hline Tratamentos salinos $\left(\mathrm{mM} \mathrm{de} \mathrm{CaCl}_{2}\right)$ & $\mathrm{PA}(\mathrm{cm})$ & $\mathrm{CR}(\mathrm{cm})$ & $\mathrm{AT}(\mathrm{cm})$ & $\mathrm{NF}(\mathrm{cm})$ & $\mathrm{DC}(\mathrm{cm})$ & $\mathrm{PMSPA}$ & $\mathrm{PMSR}$ & $\mathrm{PMST}$ \\
\hline 0 & $14,14 \mathrm{a}$ & $10,25 \mathrm{a}$ & $24,39 \mathrm{a}$ & $2,30 \mathrm{a}$ & $3,99 \mathrm{a}$ & $0,68 \mathrm{a}$ & $2,16 \mathrm{a}$ & $2,84 \mathrm{a}$ \\
25 & $7,81 \mathrm{~b}$ & $4,72 \mathrm{~b}$ & $12,79 \mathrm{~b}$ & $1,83 \mathrm{~b}$ & $3,49 \mathrm{a}$ & $0,15 \mathrm{~b}$ & $0,36 \mathrm{~b}$ & $0,57 \mathrm{~b}$ \\
50 & $1,12 \mathrm{c}$ & $0,68 \mathrm{c}$ & $1,81 \mathrm{c}$ & $0 \mathrm{c}$ & $1,30 \mathrm{~b}$ & $0,00 \mathrm{c}$ & $0,02 \mathrm{c}$ & $0,02 \mathrm{c}$ \\
75 & $0,55 \mathrm{c}$ & $0,30 \mathrm{c}$ & $0,85 \mathrm{c}$ & $0 \mathrm{c}$ & $1,02 \mathrm{~b}$ & $0,00 \mathrm{c}$ & $0,01 \mathrm{c}$ & $0,01 \mathrm{c}$ \\
100 & $0,17 \mathrm{c}$ & $0,15 \mathrm{c}$ & $0,33 \mathrm{c}$ & $0 \mathrm{c}$ & $0,40 \mathrm{~b}$ & $0,00 \mathrm{c}$ & $0,01 \mathrm{c}$ & $0,01 \mathrm{c}$ \\
\hline $\mathrm{CV}(\%)$ & 19,02 & 17,72 & 15,73 & 19,09 & 11,92 & 33,28 & 10,66 & 9,91 \\
\hline
\end{tabular}

*Médias seguidas da mesma letra na coluna, não diferem estatisticamente entre si pelo teste de Tukey a $5 \%$ de probabilidade. CV _ Coeficiente de variação.

Reduções lineares na parte aérea e na raiz em função da diminuição do potencial osmótico pelo acréscimo da salinidade, foram observadas por Dutra et al. (2014) em plântulas de Jacaranda pteroides. Assim como em plântulas de Guizotia abyssinica, com o incremento do CaCl2 (Gordim et al., 2012). A diminuição do crescimento é um dos primeiros efeitos da salinidade, provocado pela diminuição da expansão celular devido a não absorção de água, bem como resultado do efeito de toxicidade dos íons que foram absorvidos e são acumulados no protoplasma (Ferreira; Borghetti, 2004). Nas raízes, assim como nas folhas, a redução do crescimento após a indução do estresse pode ser uma consequência das alterações nas relações hídricas da célula, com consequente comprometimento na divisão celular (Munns et al., 2002).

Verificou-se efeito negativo da salinidade também para 0 número de folhas, onde 0 tratamento de $25 \mathrm{mM}$ apresentou uma redução de cerca de $20 \%$, enquanto as concentrações salinas de 50 a 100 mM não apresentaram emissão de folhas. Houve efeito negativo da salinidade também para o diâmetro, o qual começou a reduzir significativamente a partir de $50 \mathrm{mM}$ de $\mathrm{CaCl}_{2}(67,41 \%)$ e os níveis 75 e $100 \mathrm{mM}$ reduziu em até $90 \%$, em relação ao controle (Tabela 3 ).

Plântulas de Cedrella odorota também tiveram seu comprimento da parte aérea e da raiz reduzidos pelo 
aumento do nível de $\mathrm{CaCl}_{2}$, sendo afetado a partir do nível de $25 \mathrm{mM}$ (Ferreira et al., 2013). Os mesmos resultados foram observados para as espécies de Albizia lebbeck (LIMA et al., 2015), Mimosa caesalpiniifolia (Ribeiro et al., 2008) e Caesalpinia ferrea (Freitas et al., 2010), em que à medida que houve aumento da concentração dos sais no substrato, os comprimentos das plântulas foram reduzidos significativamente. Nunes et al., (2009) observaram que $0 \mathrm{CaCl}_{2}$ foi a fonte salina que menos afetou o desenvolvimento inicial de plântulas de Crotalaria juncea quando comparado com o $\mathrm{NaCl}$ e $\mathrm{KCl}$.

O efeito osmótico induz à deficiência hídrica nas plantas, provocando alterações morfológicas e anatômicas. Dentre as mudanças morfológicas, destaca-se a redução no diâmetro do caule, pois 0 estresse salino causa um rápido decréscimo da taxa de crescimento foliar. A queda na velocidade de elongação foliar resulta de uma redução no número de células em processo de divisão celular (Willadino; Camara, 2010).

0 acréscimo da salinidade reduziu significativamente 0 peso da matéria seca da parte aérea, raiz e total (Tabela 3), onde se constatou que à medida que os níveis de salinidade aumentaram, houve diminuição da massa seca de plântulas de mulungu. Para o nível de $25 \mathrm{mM}$ de $\mathrm{CaCl}_{2}$, a redução foi de aproximadamente $80 \%$ para a parte aérea, raiz e total. Enquanto para os níveis de 50,75 e $100 \mathrm{mM}$ de $\mathrm{CaCl}_{2}$ esta redução foi cerca de $99 \%$, uma vez que as estruturas das plântulas não se desenvolveram.

Diversas espécie apresentaram um menor peso da matéria fresca ao germinarem sob substratos salinos, tais como, Cnidosculus phyllacanthus (Silva et al., 2005), Moringa oleifera (Elhag et al. 2012; Oliveira et al., 2009), Delonix regia (NOGUEIRA et al., 2012) e Cedrella odorata (Ferreira et al., 2013).

Nas raízes, assim como nas folhas, a redução do crescimento imediatamente após a indução do estresse é consequência de alterações nas relações hídricas da célula. 0 excesso de íons próximo à raiz provoca efeitos deletérios no crescimento, pois é necessário um maior gasto de energia para que a água seja absorvida (Medeiros et al., 2007).

Guimarães et al., (2013), ao avaliarem o efeito de diferentes condutividades elétricas $(0,5 ; 2,5 ; 5,0 \mathrm{e}$ $\left.7,5 \mathrm{dS} . \mathrm{m}^{-1}\right)$ obtidas com a adição de $\mathrm{NaCl}$ em água, em sementes de Erythrina velutina, constataram que a emergência da plântula, o índice de velocidade de emergência, altura de plântulas, número de folhas, comprimento da raiz principal e massa da matéria seca da plântula, foram afetados a partir do segundo tratamento testado $\left(2,5 \mathrm{dS} \cdot \mathrm{m}^{-1}\right)$. Os resultados obtidos na presente pesquisa corroboram com 0 respectivo autor, uma vez que ao nível de $25 \mathrm{mM}$ de $\mathrm{CaCl}_{2}(3,1$ $\left.\mathrm{dS} . \mathrm{m}^{-1}\right)$, todas as variáveis avaliadas foram afetadas, demonstrando a sensibilidade da espécie à salinidade induzida pelo $\mathrm{CaCl}_{2}$.

Desta forma, conclui-se que o aumento da salinidade daágua de irrigação afeta de forma prejudicial, o processo de emergência e o desenvolvimento inicial de mulungu a partir da concentração de $50 \mathrm{mM}$ de $\mathrm{CaCl}_{2}$. Sementes de E. velutina são sensíveis à salinidade e toleram a concentração salina de até 25 $\mathrm{mM}$ de $\mathrm{CaCl}_{2}$.

\section{AGRADECIMENTOS}

Ao Centro de Referência para Recuperação de Áreas Degradadas (CRAD/UNIVASF) pela doação das sementes para a realização da pesquisa.

\section{REFERÊNCIAS BIBLIOGRÁFICAS}

Andréo-Souza, Y.; Pereira, A.L.; Silva, F.F.S.; RibeiroReis, R.C.; Evangelista, M.R. V.; Castro, R.D.; Dantas, B.F. Efeito da salinidade na germinação de sementes e no crescimento inicial de mudas de pinhão-manso. Revista Brasileira de Sementes, 2010, 32, 2, 83-92.

Azcón-Aguilar, C.; Encina, C.L.; Azcón, R.; Barea, J.M. Mycotrophy of Annona-cherimola and the morphology of its mycorrhizae. Mycorrhiza, 1994, 4, 161-168.

Barreto, H.B.F.; Freitas, R.M.O.; Oliveira, L.A.A.; Araújo, J.A.M.; Costa, E.M. Efeito da irrigação com água salina na germinação de sementes de sábia (Mimosa caesalpiniifolia Benth). Revista Verde, 2010, 5, 3, 125-130.

Barros, M.F.C.; Fontes, M.P.F.; Alvarez, V.H.; Ruiz, H.A. Recuperação de solos afetados por sais pela aplicação de gesso de jazida e calcário no Nordeste do Brasil. Revista Brasileira Engenharia Agrícola e Ambiental, 2004, 8, 59-64.

Carvalho, P.E.R. Mulungu (Erythrina velutina). Colombo-PR: Embrapa Florestas, 2008. 8p. (Circular técnica, 160). 
D’almeida, D.M.B.A.; Andrade, E.M.; Meireles, A.C.M.; Ness, R.L. Importância relativa dos íons na salinidade de um Cambissolo na Chapada do Apodi, Ceará. Engenharia Agrícola, 2005, 25, 3, 615-621.

Dias, N.D.; Blanco, F.F. Efeitos dos sais no solo e na planta. In: Gheyi, H.R.; DIAS, N.S.; Lacerda, C.F. Manejo da salinidade na agricultura: Estudos básicos e aplicados. Fortaleza: Instituto Nacional de Ciência e Tecnologia em Salinidade, 2010, 129-140.

Dutra, T.R.; Massad, M.D.; Matos, P.S.; Oliveira, J.C.; Sarmento, M.F. Q. Germinação e crescimento inicial de plântulas de carobinha-do-campo submetido ao estresse hídrico e salino. Agropecuária Científica no Semiárido, 2014, 10, 4, 39-45.

Elhag, A.Z.; Abdalla, M.H. Effect of Sodium Chloride on Germination and Emergence of Moringa (Moringa oleifera L.) Seeds. Journal of Science and Technology, 2012, 13, 2, 62-67.

Ferreira, A.G.; Borguetti, F. Germinação: do básico ao aplicado. Porto Alegre: Artmed, 2004.

Ferreira, E.G.B.S.; Matos, V.P.; Sena, L.H.M.; Oliveira, R.G.; Sales, A.G.F.A. Processo germinativo e vigor de sementes de Cedrela odorata L. sob estresse salino. Ciência Florestal, 2013, 23, 1, 99-105.

Freitas, R.M.O.; Nogueira, N.W.; Oliveira, F.N.; Costa, E.M.; Ribeiro, M.C.C. Efeito da irrigação com água salina na emergência e crescimento inicial de plântulas de jucá. Revista Caatinga, 2010, 23, 3, 54-58.

Gonçalves, L.O.; Pinheiro, J.B.; Zucchi, M.I.; Mann, R.S. Caracterização genética de mulungu (Erythrina velutina Willd.) em áreas de baixa ocorrência. Revista Ciência Agronômica, 2014, 45, 2, 290-298.

Gordin, C.R.B.; Marques, R.F.; Masetto, T.E.; Souza, L.C.F. Estresse salino na germinação de sementes e desenvolvimento de plântulas de niger (Guizotia abyssinica (L.f.) Cass.). Acta Botânica Brasílica, 2012, 26, 4, 966-972.

Guimarães, I.P.; Vieira, F.E.R.; Torres, S.B.; Oliveira, F.N. Efeito da salinidade da água de irrigação na emergência e crescimento inicial de plântulas de mulungu. Revista Brasileira de Ciências Agrárias, 2013, 8, 1, 137-142.

Holanda, A.C.; Santos, R.V.; Souto, J.S.; Alves, A.R. Desenvolvimento inicial de espécies arbóreas em ambientes degradados por sais. Revista de Biologia e Ciências da Terra, 2007, 7, 1.

Lima, B.G.; Torres, S.B. Estresses hídrico e salino na germinação de sementes de Zizyphus joazeiro Mart. (Rhamnaceae). Revista Caatinga, 2009, 22, 4, 93-99.

Lima, M.F.P.; Porto, M.A.F.; Torres, S.B.; Freitas, R.M.O.; Nogueira, N.W.; Carvalho, D.R. Emergência e crescimento inicial de plântulas de albízia submetidas à irrigação com água salina. Revista Brasileira de Engenharia Agrícola e Ambiental, 2015, 19, 2, 106-112.

Lopes, J.C.; Macedo, C.M.P. Germinação de sementes de couve chinesa sob influência do teor de água, substrato e estresse salino. Revista Brasileira de Sementes, 2008, 30, 3, 79-85.

Maguire, J.D. Speed of germination: aid in selection and evaluation for seedling emergence and vigour. Crop Science, 1962, 2, 2, 176-177.

Marcos Filho, J. Fisiologia de sementes de plantas cultivadas. Londrina: ABRATES, 2013, 12, 659p.

Medeiros, J.F.; Silva, M.C.C.; Sarmento, D.H.A.; Barros, A.D. Crescimento do meloeiro cultivado sob diferentes níveis de salinidade, com e sem cobertura do solo. Revista Brasileira de Engenharia Agrícola e Ambiental, 2007, 11, 248-255.

Munns, R.; Husain, S. Rivelli, A.R.; Richard, A.J.; Condon, A.G.; Megan, P.L.; Evans, S.L.; Schachtman, D.P.; Hare, R.A. Avenues for increasing salt tolerance of crops, and the role of physiologically based selection traits. Plant and Soil, 2002, 247, 93-105.

Nogueira, N.W.; Lima, J.S.S.; Freitas, R.M.O, Ribeiro, M.C.C.; Leal, C.C.P.; Pinto, J. R. S. Efeito da salinidade na emergência e crescimento inicial de plântulas de flamboyant. Revista Brasileira de Sementes, 2012, 34, 3, 466-472.

Nunes, A.S.; Lourenção, A.L.F.; Pezarico, C.R.; Scalon, 
S.P.Q.; Gonçalves, M.C. Fontes e níveis de salinidade na germinação de sementes de Crotalaria juncea $\mathrm{L}$. Ciência e Agrotecnologia, 2009, 33, 3, 753-757.

Oliveira, F.R.A.; Oliveira, F.A.; Guimarães, I.P.; Medeiros, J.F.; Oliveira, M.K.T.; Freitas, A.V.L; Medeiros, M. A. Emergência de plântulas de moringa irrigada com água de diferentes níveis de salinidade. Bioscience Journal, 2009, 25, 66-74.

Ribeiro, M.C.C.; Barros, N.M.S.; Barros Júnior, A. P.; SILVEIRA, L. M. Tolerância do sabiá (Mimosa caesalpiniifolia benth) à salinidade durante a germinação e o desenvolvimento de plântulas. Revista Caatinga, 2008, 21, 5, 123-126.

Schossler, T.R.; Machado, D.M.; Zuffo, A.M.; Andrade, F.R.; Piauilino, A.C. Salinidade: efeitos na fisiologia e na nutrição mineral de plantas. Enciclopédia Biosfera, 2012, 8, 15, 1563-1578.
Silva, M.B.R.; Batista, R.C.; Lima, V.L.A.; Barbosa, E.M.; Barbosa M.F.N. Crescimento de plantas jovens da espécie florestal favela (Cnidosculus phyllacanthus Pax \& K. Hoffmem) em diferentes níveis de salinidade da água. Revista de Biologia e Ciências da Terra, 2005, 5, 1-13.

Vasconcelos, R.R.A.; Barros, M.F.C.; Silva, E.F.F.; Graciano, E.S.A.; Fontenele, A. J.P.B.; Silva, N.M.L. Características físicas de solos salino-sódicos do semiárido pernambucano em função de diferentes níveis de gesso. Revista Brasileira de Engenharia Agrícola e Ambiental, 2013, 17, 12, 1318-1325.

Willadino, L.; Camara, T. R. Tolerância das plantas à salinidade: aspectos fisiológicos e bioquímicos. Enciclopédia biosfera, 2010, 6, 11, 1-23. 
